\title{
Composition and Mineralogy of Cometary Dust
}

\author{
Martha S. Hanner \\ Jet Propulsion Laboratory/California Institute of Technology \\ John P. Bradley \\ Lawrence Livermore Laboratory
}

\begin{abstract}
Cometary dust is an unequilibrated, heterogeneous mixture of crystalline and glassy silicate minerals, organic refractory material, and other constituents such as iron sulfide and possibly minor amounts of iron oxides. Carbon is enriched relative to CI chondrites; some of the $\mathrm{C}$ is in an organic phase. The silicates are Mg-rich, while iron is distributed in silicates, sulfides, and FeNi metal. Infrared spectra of silicate emission features in Comet Hale-Bopp have led to identification of the minerals forsterite and enstatite. The strong similarity of all known cometary dust properties to the anhydrous chondritic aggregate class of interplanetary dust particles (IDPs) argues that comets are the source of these IDPs. High D/H ratios in organic refractory material in these IDPs as well as the physical and chemical structure of glassy silicate grains suggests a presolar origin for at least some components of cometary dust.
\end{abstract}

\section{INTRODUCTION}

Comets contain some of the least-altered material surviving from the early solar nebula. Cometary dust may contain both presolar particulates and solar nebula condensates. Their structure and mineralogy may hold important clues about the chemical and physical processes in the early solar system. Radial gradients in the temperature and chemical composition of the solar nebula and the extent of mixing of material between the warm inner regions and cold outer regions of the nebula at the epoch of comet formation should be evident today as differences in dust properties among comets related to their place of origin.

The extensive infrared spectroscopy of Comet HaleBopp, from the ground and from ESA's Infrared Space Observatory, has led to a revolution in our understanding of the silicate mineralogy in comets. In parallel, the past decade has seen extrodinary advances in the analysis of interplanetary dust particles (IDPs), leading to the discovery of eroded, glassy silicates of likely interstellar origin and isotopic anomalies in silicate and carbonaceous components also pointing toward a possible presolar origin.

This chapter summarizes our present knowledge of the composition and mineralogy of cometary dust based on in situ sampling during the spacecraft encounters with Comet 1P/Halley in 1986, Earth-based remote sensing via infrared spectroscopy, and laboratory analysis of captured IDPs of probable cometary origin.

\section{IN SITU SAMPLING}

The most direct means of determining the composition of cometary dust is by in situ sampling or analysis of returned samples. To date, in situ sampling has been carried out for just one comet, 1P/Halley. The two Soviet Vega spacecraft and ESA's Giotto probe each carried an impact ionization time of flight mass spectrometer to measure the elemental composition of the dust (Kissel et al., 1986a,b). The composition was recorded for about 5000 particles in the mass range $10^{-16}-10^{-11} \mathrm{~g}$, sampled over tens of thousands of kilometers in the coma along the three trajectories.

The sampled particles divide into three main types: mass spectra dominated by the major rock-forming elements, $\mathrm{Mg}$, $\mathrm{Si}, \mathrm{Ca}, \mathrm{Fe}$; mass spectra consisting primarily of the light elements $\mathrm{H}, \mathrm{C}, \mathrm{N}, \mathrm{O}$, the "CHON" particles; and mixed spectra containing both the rock and $\mathrm{CHON}$ elements. If one defines mixed particles as having a ratio of $\mathrm{C}$ to rock-forming elements between 0.1 and 10 , then $~ 50 \%$ of the particles are mixed and $\sim 25 \%$ are rock and CHON respectively (Fomenkova et al., 1992). At some level, however, the CHON and rocky material are mixed down to the finest submicrometer scale in all particles (Lawler and Brownlee, 1992). The bulk abundances of the major rock-forming elements appear to be solar (chondritic) within a factor of 2 (Jessberger et al., 1988; Jessberger, 1999). (Conversion of the mass spectra to relative elemental abundances depends on the ion yields, which are uncertain by at least a factor of 2, because the instrument could not be calibrated at the high flyby speed of $\sim 70 \mathrm{~km} / \mathrm{s}$.) The $\mathrm{C}$ abundance is roughly 10 times that of the primitive $\mathrm{CI}$ chondrites.

The rocky material displays a wide range in $\mathrm{Fe} / \mathrm{Mg}$ abundance, but a narrow range in Si/Mg (Lawler et al., 1989). Magnesium-rich (Fe-poor) silicates comprise at least $40 \%$ and perhaps $\geq 60 \%$ of the rocky particles (Jessberger, 1999). Iron is present in other minerals including metals (1-2\%), iron sulfides $(\sim 10 \%)$, and possibly iron oxide $(\leq 1 \%)$ (Schulze et al., 1997).

The CHON spectra are evidence for an organic refractory component in the cometary dust. Significant clustering of subgroups (e.g., spectra dominated by $[\mathrm{H}, \mathrm{C}],[\mathrm{H}$, 
$\mathrm{C}, \mathrm{O}$, etc.) indicate variable composition of the organic refractory material (Fomenkova et al., 1994).

Isotopic ratios were found to be solar, within the measurement uncertainties, with the exception of ${ }^{12} \mathrm{C} /{ }^{13} \mathrm{C}$ (Jessberger, 1999). While low ratios ( ${ }^{13} \mathrm{C}$ enrichment) were judged to be uncertain due to noise of uncertain origin, Jessberger (1999) reported that definite ${ }^{12} \mathrm{C}$ enrichments, up to ${ }^{12} \mathrm{C} /{ }^{13} \mathrm{C}$ 5000 , were identified, indicative of presolar nucleosynthesis products.

\section{SPECTROSCOPY OF COMETARY SILICATES}

Small silicate grains in the cometary dust coma will produce an emission feature near $10 \mu \mathrm{m}$ due to stretching vibrations in $\mathrm{Si}-\mathrm{O}$ bonds. Additional bending mode vibrations occur between 16 and $35 \mu \mathrm{m}$. The wavelengths and shapes of these features are diagnostic of the mineral composition. The $10-\mu \mathrm{m}$ feature lies within the $8-13-\mu \mathrm{m}$ atmospheric "window," allowing groundbased observations. Although some 20- $\mu \mathrm{m}$ observations can also be made from the ground, the full $16-35-\mu \mathrm{m}$ region is best studied from above the atmosphere.

Low-resolution $(R \sim 50-100), 8-13-\mu \mathrm{m}$ spectra now exist for a number of comets. Several comets display strong structured silicate emission with total flux/continuum at $10 \mu \mathrm{m}>1.5$. These include long-period comets Bradfield (1987 XXIX = C/1987 P1) (Hanner et al., 1990, 1994a), Levy (1990 XX = C/1990 K1) (Lynch et al., 1992), Hyakutake (C/1996 B2), and Hale-Bopp (C/1995 O1) (Hanner et al., 1999; Wooden et al., 1999; Hayward et al., 2000); new comet Mueller (1994 I = C/1993 A1) (Hanner et al., 1994b); and 1P/Halley (Bregman et al., 1987; Campins and Ryan, 1989).

By far the strongest silicate emission was seen in HaleBopp. This comet was also unusual in displaying a strong silicate feature even at 4.6 AU preperihelion (Crovisier et al., 1996; Grün et al., 2001). Near-perihelion, groundbased spectra were obtained by several groups; all spectra show similar structure (see Hanner et al., 1999, for a review). A typical spectrum is presented in Fig. 1. The observed fluxes have been divided by a blackbody fitted at 8 and 12.5$13 \mu \mathrm{m}$. There are three main peaks, at 9.2, 10.0, and $11.2 \mu \mathrm{m}$, and minor structure at 11.9 and $10.5 \mu \mathrm{m}$. The spectral shape is very similar to that in P/Halley and the other comets cited above.

The 11.2- $\mu \mathrm{m}$ peak is attributed to crystalline olivine $\left([\mathrm{Mg}, \mathrm{Fe}]_{2} \mathrm{SiO}_{4}\right)$, based on the good spectral match with the measured spectral emissivity of Mg-rich olivine (Stephens and Russell, 1979; Koike et al., 1993). The 11.9- $\mu$ m shoulder is also due to crystalline olivine. If all the silicates have similar temperatures, then only a small fraction (15-20\%) of the silicate material needs to be in the form of crystalline olivine to produce the observed peak (Hanner et al., 1994a); the mass absorption coefficient of olivine near $11.2 \mu \mathrm{m}$ is a factor of 3-10 times that of glassy silicates (Day, 1976, 1981). The broader 10- $\mu$ maximum in the

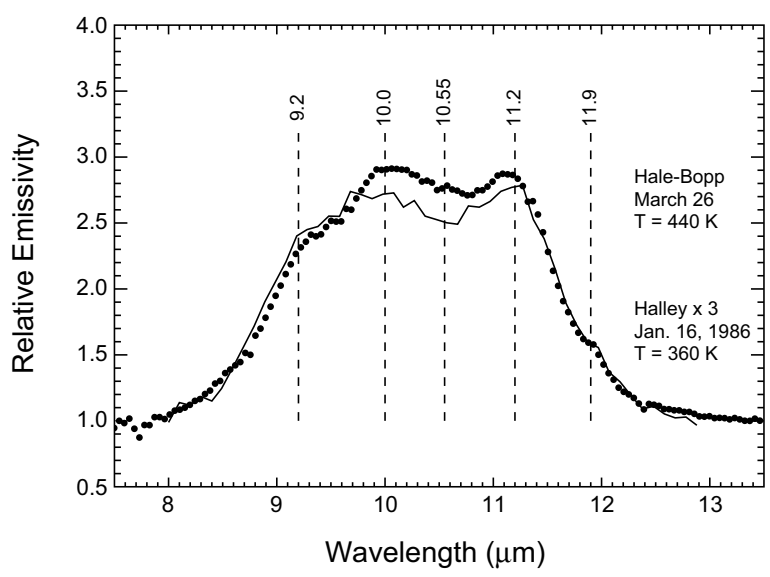

Fig. 1. The $10-\mu \mathrm{m}$ silicate emission feature in Hale-Bopp at $r=$ $0.92 \mathrm{AU}$ (dots) and 1P/Halley at $\mathrm{r}=0.79 \mathrm{AU}$ from Campins and Ryan (1989) (line). Each spectrum has been divided by a Planck function for the temperature shown and the Halley spectrum has been multiplied by 3 . The spectral peaks in the Hale-Bopp spectrum are marked. From Hanner et al. (1999).

cometary spectra is characteristic of amorphous olivine (Stephens and Russell, 1979); crystalline olivine has a secondary peak at $10 \mu \mathrm{m}$ as well.

The 9.2- $\mu \mathrm{m}$ feature, first recognized in Hale-Bopp, is a signature of pyroxene $\left([\mathrm{Mg}, \mathrm{Fe}] \mathrm{SiO}_{3}\right)$. A peak wavelength of $9.2 \mu \mathrm{m}$ corresponds to amorphous, Mg-rich pyroxene (Stephens and Russell, 1979; Dorschner et al., 1995). Crystalline pyroxenes generate more variety in their spectra (Sandford and Walker, 1985; Jaeger et al., 1998). Peaks at $10-11 \mu \mathrm{m}$ contribute to the width of the cometary feature and the structure near $10.5 \mu \mathrm{m}$. A peak near $9.3 \mu \mathrm{m}$ is generally present in crystalline pyroxenes as well.

A remarkable 16-45- $\mu$ m spectrum of Comet Hale-Bopp at $r=2.9$ AU, shown in Fig. 2, was acquired with the shortwavelength spectrometer (SWS) on ESA's Infrared Space Observatory (Crovisier et al., 1997, 2000). Five peaks are clearly visible, corresponding in every case to laboratory spectra of crystalline forsterite (Mg-olivine) (Koike et al., 1993). Minor spectral structure is attributed to crystalline enstatite (Mg-pyroxene) (Wooden et al., 1999; Crovisier et al., 2000). This result is significant in indicating that the silicates are $\mathrm{Mg}$-rich, in agreement with the elemental composition detected in P/Halley's coma.

These spectra contrast with airborne spectra of Comet $\mathrm{P} /$ Halley, the only other complete $20-30-\mu \mathrm{m}$ spectra of a comet. A 16-30- $\mu \mathrm{m}$ spectrum at $\mathrm{r}=1.3 \mathrm{AU}$ (spectral resolution $0.2 \mu \mathrm{m}$ ) displays a sharp peak at $28.4 \mu \mathrm{m}$, but only weak features at 23.8 and $19.6 \mu \mathrm{m}$ (Herter et al., 1987). Twenty- to $35-\mu \mathrm{m}$ spectra with $0.5-1-\mu \mathrm{m}$ resolution, taken at 1.2-AU preperihelion and 1.4-AU postperihelion, show only weak excess above the continuum at 24 and $33 \mu \mathrm{m}$ (Glaccum et al., 1987). This result is puzzling. Although the strength of the $10-\mu \mathrm{m}$ silicate feature was quite variable from day to day, an 11.2- $\mu$ m olivine peak was clearly 


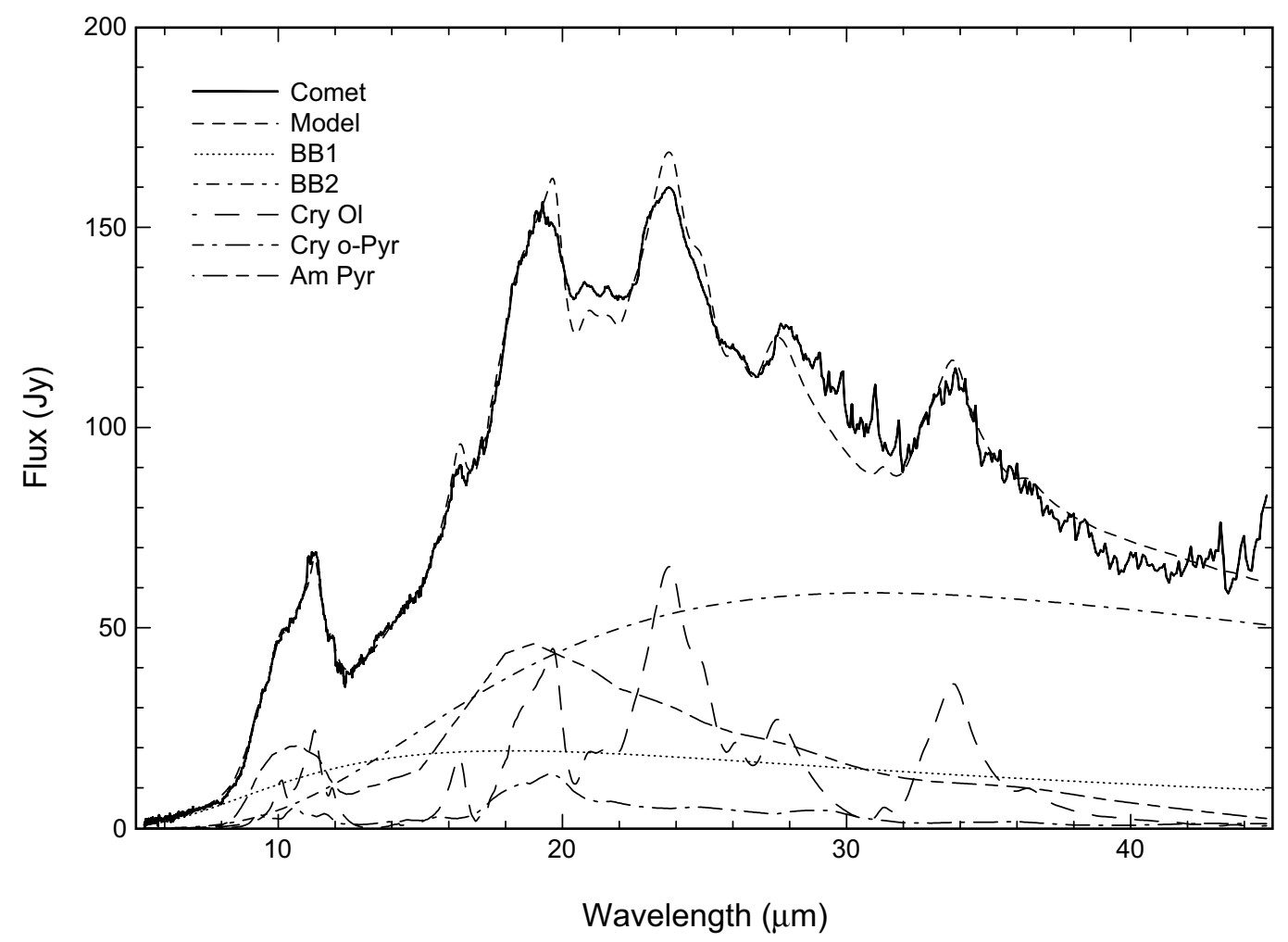

Fig. 2. ISO SWS spectrum of Comet Hale-Bopp at $\mathrm{r}=2.8 \mathrm{AU}$, degraded to $\mathcal{R}=500$, compared with a five-component dust model: $280 \mathrm{~K}$ blackbody (BB1); $165 \mathrm{~K}$ blackbody (BB2); forsterite (Cry Ol 22\%); orthopyroxene (Cry o-Pyr 8\%); and amorphous pyroxene (Am Pyr 70\%). From Crovisier et al. (2000).

visible as part of a strong $10-\mu \mathrm{m}$ feature on the same day as the 1.2-AU airborne spectrum (Bregman et al., 1987). Viewing restrictions on solar elongation angle prevented SWS observations when Hale-Bopp was near $1 \mathrm{AU}$, so we do not know how the 16-35- $\mu$ m spectrum evolved as the grains were heated. However, an SWS spectrum acquired at 3.9-AU postperihelion still displayed the major forsterite peaks (Crovisier et al., 2000).

To produce a strong emission feature, silicate particles must have radii on the order of $1 \mu \mathrm{m}$ or smaller. Larger particles will display a feature only if they are very porous aggregates and the individual constituent grains are micrometer-sized or smaller.

Spectral models to match the Hale-Bopp spectra with a mixture of silicate minerals have been presented by Brucato et al. (1999), Colangeli et al. (1999), Galdemard et al. (1999), Hayward et al. (2000), Wooden et al. (1999, 2000), and Harker et al. (2002). Wooden et al. (1999, 2000) proposed that the observed changes in spectral shape with heliocentric distance can be explained by temperature differences between more-transparent (cooler) Mg-rich pyroxene grains and less-transparent (warmer) olivine grains. In their model, the cooler crystalline pyroxenes comprise the major fraction of small silicate grains $(\sim 90 \%)$, and these grains produce the enhanced $9.3-\mu \mathrm{m}$ feature near perihelion. Hayward et al. (2000) assumed in their modeling that all the silicate grains would have sufficient thermal contact with absorbing material to be warm, regardless of their $\mathrm{Mg}$ / Fe content. They concluded that glassy pyroxenes were the most abundant component $(>40 \%)$ and that crystalline olivine comprised $\leq 20 \%$ of the small silicate grains.

Harker et al. (2002) modeled the combined 8-13- $\mu \mathrm{m}$ spectra, IR photometry, and SWS spectra from October 1996 ( $r=2.8 \mathrm{AU})$; the extended wavelength baseline enabled them to restrict the temperatures for the various amorphous and crystalline silicate components (tied to their $\mathrm{Mg}$ / Fe abundance ratios). They introduced porosity of the $\mathrm{C}$ and amorphous silicate particles (modeled with effective medium theory) by adopting the fractal dimension, D, as one of the model parameters. By comparing their best-fit model at $r=2.8$ AU with the $19978-13-\mu m$ spectra and photometry, they concluded that the size distribution steepened and fractal dimension decreased as the comet approached perihelion. Crystalline silicates constituted about $30 \%$ by mass of the small dust grains in the coma at all epochs.

In summary, the observed spectral features imply a complex mineralogy for the cometary silicates, including both amorphous and crystalline grains of pyroxene and olivine composition. This mineralogy is consistent with the chondritic aggregate IDPs, described in the next section.

Not all comets display strong $10-\mu \mathrm{m}$ emission features. The spectra of four new comets discussed in Hanner et al. 
(1994a) are puzzling; each has a unique spectrum that is not yet understood. For example, an extremely broad emission feature was present in Wilson $(1987$ VII $=$ C/1986 P1) (Lynch et al., 1989), suggesting a very amorphous silicate material. It is possible that we are witnessing the effect of cosmic-ray damage to the outermost layer of the nucleus over the lifetime of the Oort cloud.

No strong $10-\mu \mathrm{m}$ emission feature has yet been seen in a short-period comet. Broad emission features about $20 \%$ above a black-body continuum were present in spectra of Comets 4P/Faye and 19P/Borrelly (Hanner et al., 1996) and 103P/Hartley 2 (Crovisier et al., 2000). Filter photometry of $81 \mathrm{P} /$ Wild 2 revealed a feature about $25 \%$ above the continuum; no spectrum exists (Hanner and Hayward, 2003). The ratio of the flux in a narrow $10.4-\mu \mathrm{m}$ filter to the flux in a broad $10-\mu \mathrm{m}(\mathrm{N})$ filter indicated some silicate emission in Comets P/Encke, P/Stephan-Oterma, and P/Tuttle (Campins et al., 1982). Gehrz et al. (1989) did not detect silicate emission in P/Encke near perihelion in 1987. Other short-period comets with $10-\mu \mathrm{m}$ filter photometry show no feature at the $10 \%$ level. The absence of strong silicate emission in the short-period comets could be explained either by a difference in the composition between Oort cloud and Kuiper belt comets or by a lower abundance of submicrometer-sized particles. Short-period comets generally have been outgassing during many orbits in the inner solar system and the smaller or more fluffy particles may have been preferentially expelled over time. Thus, a lower abundance of isolated small grains or very fluffy aggregates of small grains in the coma is the simplest explanation for the lack of a strong silicate feature, although compositional differences cannot be ruled out.

\section{INTERPLANETARY DUST PARTICLES FROM COMETS}

The submicrometer grain size, high $\mathrm{Mg} / \mathrm{Fe}$ ratio, and mix of crystalline and noncrystalline olivine and pyroxenes in the cometary dust have no counterpart in any meteoritic material, with the exception of the anhydrous chondritic aggregate IDPs. These are fine-grained heterogeneous aggregates having chondritic abundances of the major rockforming elements; they comprise a major fraction of the IDPs captured in the stratosphere (Fig. 3). Typical grain sizes within the aggregates are $0.1-0.5 \mu \mathrm{m}$; micrometersized crystals of forsterite and enstatite are also present (Bradley et al., 1992). These aggregate IDPs are thought to originate from comets, based on their porous structure, small grain size, high $\mathrm{C}$ content, and relatively high atmospheric entry speeds. Measured He release temperatures indicate that many of these IDPs entered the atmosphere at speeds $>16 \mathrm{~km} / \mathrm{s}$, consistent with cometary orbits (Nier and Schlutter, 1993). The match between the mineral identifications in the Hale-Bopp spectra and the silicates seen in the IDPs strengthens the link between comets and this class of IDPs. Thus, the composition and structure of the porous aggregate IDPs can be used to augment our understanding of the composition and origin of cometary dust.

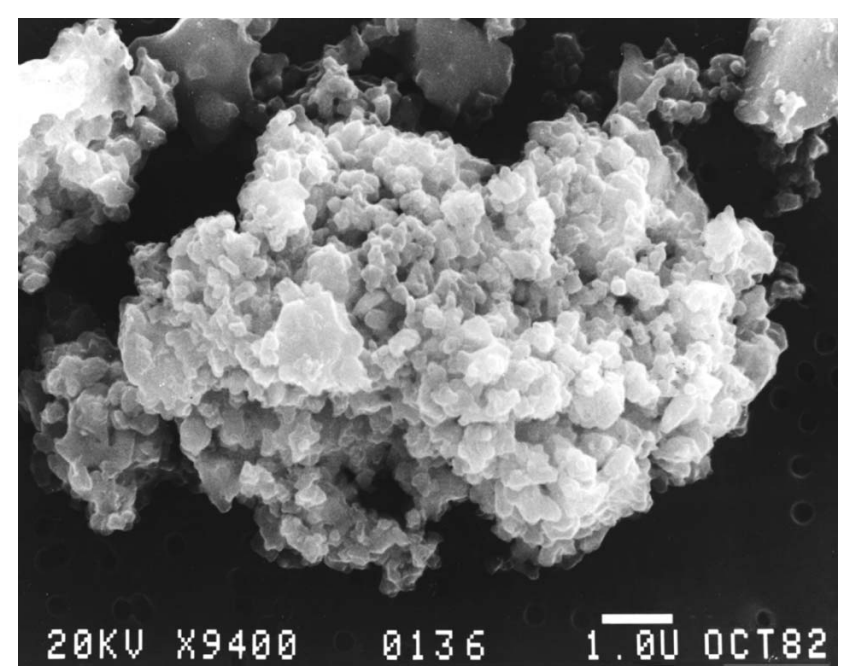

Fig. 3. Secondary electron micrograph of a chondritic porous (CP) interplanetary dust particle. Bar is $1 \mu \mathrm{m}$ in length. The fragile microstucture and high porosity of this particle are consistent with a cometary origin. The submicrometer-sized grains are mainly GEMS and carbonaceous material. The angular, micrometer-sized components include single crystals of forsterite, enstatite, and FeNi sulfides.

The major form of noncrystalline silicates in the chondritic aggregate IDPs are the glass with embedded metal and sulfides (GEMS). These are submicrometer-sized glassy $\mathrm{Mg}$-silicate grains with embedded nanometer $\mathrm{FeNi}$ and $\mathrm{Fe}$ sulfide crystals (Bradley, 1994). The GEMS show evidence of exposure to large doses of ionizing radiation (e.g., Demyk et al., 2001, Carrez et al., 2002), such as eroded surfaces, $\mathrm{O}$ enrichment, gradients in $\mathrm{Mg} / \mathrm{Si}$ ratio decreasing outward from the center, and formation of reduced FeNi metal, pointing to exposure in the interstellar medium prior to their incorporation into comets. Some GEMS contain heavily etched relict mineral grains of sulfides or Mg-rich silicate crystals. The physical and chemical properties of GEMS are similar to those inferred for interstellar grains.

Infrared spectra of GEMs-rich IDP samples (thin sections containing GEMS and silicate crystals) resemble the cometary silicate spectra, while spectra of individual GEMS reveal a single broad peak near $9.3 \mu \mathrm{m}$ (glassy pyroxene) or $9.8 \mu \mathrm{m}$ (glassy olivine) (Bradley et al., 1999). The peak wavelength, width, and long-wavelength asymmetry of the 9.8- $\mu \mathrm{m}$ peak are similar to the interstellar silicate feature, such as that of the Trapezium.

Detection of nonsolar isotopic abundances in GEMS would constitute strong evidence for an interstellar origin. GEMS are frequently embedded in a carbonaceous material that displays high $\mathrm{D} / \mathrm{H}$ ratios and ${ }^{15} \mathrm{~N}$ enrichment (Keller et al., 2000). A bulk O-isotopic measurement of a GEMSrich IDP yielded the highest "whole-rock" ${ }^{16} \mathrm{O}$ enrichment yet measured in any chondritic object (Engrand et al., 1999); silicates are the main carrier of O. NanoSIMS techniques are now reaching the sensitivity to allow isotopic measurements on individual submicrometer-sized grains in 
IDPs. In early results utilizing nanoSIMS, two GEMS with nonsolar O-isotopic ratios have been detected (Messenger et al., 2003).

The crystalline silicates found in chondritic porous IDPs are primarily the $\mathrm{Mg}$-rich minerals forsterite and enstatite. This mineralogy is consistent with the $8-35-\mu \mathrm{m}$ spectrum of Hale-Bopp and the in situ Halley elemental composition data. Grain sizes range from $\sim 0.05$ to $5 \mu \mathrm{m}$ although most are $0.1-0.75 \mu \mathrm{m}$ in diameter. Some enstatite whiskers, rods, and platelets have unusual growth patterns, such as axial screw dislocations, that indicate direct vapor phase condensation from a hot gas (Bradley et al., 1983). Some low-Fe, $\mathrm{Mn}$-enriched (LIME) enstatite and forsterite grains contain up to $5 \mathrm{wt} \% \mathrm{MnO}$, in contrast to the majority of pyroxenes and olivines in meteorites, which contain $<0.5 \mathrm{wt} . \% \mathrm{MnO}$ (Klock et al., 1989). The high Mn content is further evidence of direct condensation from a gas. At least one submicrometer forsterite grain with ${ }^{17} \mathrm{O}$ enrichment, indicating a circumstellar origin, has been identified (Messenger et al., 2003).

The chondritic aggregate IDPs have a high bulk $\mathrm{C}$ content, $2-3$ times higher on average than the primitive $\mathrm{CI}$ meteorites. Thomas et al. (1993) measured a range of 1$47 \mathrm{wt} \% \mathrm{C}$ in 100 anhydrous IDPs; in a few cases, $\mathrm{C}$ was the most abundant element by volume. The $\mathrm{C}$ is distributed throughout the IDP as a matrix surrounding the mineral grains, but not necessarily as mantles on the grains. Much of the $\mathrm{C}$ is in an organic phase, evident from X-ray absorption edge spectroscopy and $\mathrm{C}-\mathrm{H}$ stretch absorption features in the 3- $\mu \mathrm{m}$ region (Clemett et al., 1993; Flynn et al., 1999, 2000). $\mathrm{C}=\mathrm{O}$ functional groups have been identified from the absorption edge spectroscopy (Flynn et al., 2001). These results are qualitatively consistent with the nature of the CHON particles detected by the dust analysis instrument on the Halley space probes (Fomenkova et al., 1994). Carbon-rich IDPs show a red reflectance spectrum, similar to red, dark outer solar system material (Bradley et al., 1996; Keller and Messenger, 1997).

Nonsolar isotopic enrichments of $\mathrm{D} / \mathrm{H}$ and ${ }^{15} \mathrm{~N} /{ }^{14} \mathrm{~N}$ have been detected in C-rich IDPs. In particles where further analysis has been carried out, the $\mathrm{D}$ and ${ }^{15} \mathrm{~N}$ anomalies are associated with an organic carrier (e.g., Aleon et al., 2002). These isotopic enrichments are attributed to mass fractionation during low-temperature ion-molecular reactions in cold, dense interstellar molecular clouds. In some cases, the measured $\mathrm{D} / \mathrm{H}$ ratio approaches that observed in molecular clouds (Messenger, 2000). High D/H ratios, roughly twice the terrestrial value and 10 times the protosolar value, have been observed in gas phase cometary $\mathrm{H}_{2} \mathrm{O}$ and $\mathrm{HCN}$ (Meier and Owen, 1999). Iron-nickel sulfide grains are the major carrier of $S$ in the chondritic aggregate IDPs. The sulfide mineralogy is significantly different from that in primitive chondritic meteorites (Dai and Bradley, 2001). Keller et al. (2002) have proposed that sulfides are responsible for a broad $\lambda \sim 23-\mu \mathrm{m}$ feature detected around young and old stars by ISO. Laboratory spectra of pyrrhotite grains from IDPs display a broad Fe-S stretch feature centered at $\sim 23.5 \mu \mathrm{m}$, similar to the circumstellar feature. Iron sulfide was an iden- tified component of the dust in Comet Halley sampled during the spacecraft encounters (Schulze et al., 1997).

\section{ORIGINS OF COMETARY DUST}

It is clear from the in situ and remote observations of comets and the analysis of probable cometary IDPs that cometary dust is an unequilibrated, heterogeneous mixture of minerals, including both high- and low-temperature condensates. These various components do not necessarily share a common origin. Temperatures in the solar nebula beyond $5 \mathrm{AU}$, where the comet nuclei accreted, were never higher than about $160 \mathrm{~K}$, too low for significant processing of dust particles (Boss, 1994, 1998, 2004). Thus interstellar grains present in the outer solar nebula could have been preserved in comets. Material that condensed within the solar nebula also may have accreted into the comet nuclei.

The glassy silicate grains (GEMS) described in section 4 appear to constitute the major fraction of the noncrystalline silicates in cometary IDPs and the evidence is quite strong that these are interstellar grains, based on their morphology, physical and chemical structure, and inferred high radiation dosage. They are often embedded in an organic $\mathrm{C}$ material with nonsolar $\mathrm{D} / \mathrm{H}$ and ${ }^{15} \mathrm{~N} /{ }^{14} \mathrm{~N}$ isotopic ratios. The GEMS must have formed at comparatively low temperatures and were never heated sufficiently to anneal. Thus, noncrystalline cometary silicates may be predominantly of interstellar origin.

The origin of the crystalline silicates in comets is more complex. Crystalline silicate grains can form by direct condensation from a hot gas at $\mathrm{T}=1200-1400 \mathrm{~K}$, followed by slow cooling, or by annealing of amorphous silicates at temperatures around $1000 \mathrm{~K}$ or higher (Hallenbeck et al., 1998, 2000; Brucato et al., 2002; Koike and Tsuchiyama, 1992; Fabian et al., 2000). While the enstatite whiskers and rods occasionally seen in aggregate IDPs have growth patterns indicating direct vapor phase condensation, other crystalline grains in IDPs have no distinctive structure to distinguish between direct condensation or annealing. The $\mathrm{Mg}$ crystalline minerals forsterite and enstatite are predicted from thermodynamic models to be the first to condense in a hot gas at $1200-1400 \mathrm{~K}$ and only react with $\mathrm{Fe}$ at lower temperatures. Thus, direct condensation is a natural explanation for the preponderence of $\mathrm{Mg}$-silicates in comet dust.

Grain condensation or annealing could have occurred in the hot inner solar nebula. Disk midplane temperatures $\geq 1000 \mathrm{~K}$ were reached inside about $1 \mathrm{AU}$, depending on the mass infall rate (Boss, 1998; Chick and Cassen, 1997). During the early high mass accretion phase (mass infall rate $\geq 10^{-6} \mathrm{M}_{\odot} \mathrm{yr}^{-1}$ ), this hot region could have extended to 3$4 \mathrm{AU}$ (Bell et al., 2000). However, the crystalline grains must be transported out to the region where the comets formed at 5-50 AU, and the extent of radial mixing of dust is uncertain. Bockelée-Morvan et al. (2002) have shown that turbulent diffusion in the solar nebula could be an efficient process to transport the crystalline grains from the inner nebula to the region of comet formation in timescales of a few $10^{4} \mathrm{yr}$. This process is efficient as long as the grains remain 
coupled to the gas, i.e., as long as they remain small. The timescales for grain coagulation and growth are uncertain, but could be short enough to compete with radial transport.

Alternatively, Harker and Desch (2002) have proposed that small silicate grains in the solar nebula could have been thermally annealed by passing shock waves, provided that the ambient gas density was high enough to heat the grains briefly above $1200 \mathrm{~K}$. The relation between the amount of heating and the degree of crystallinity is based on laboratory annealing experiments of Hallenbeck et al. (1998, 2000). This mechanism would be effective out to about $10 \mathrm{AU}$; at larger heliocentric distances the gas density would be too low to generate sufficient grain heating.

More study is needed, however, to understand the details of the annealing process and whether crystals with the observed mineralogy and morphology could be produced. If GEMS are representative of the amorphous silicate particles present in the solar nebula, one would expect the annealed crystals to retain the FeNi nanoparticles present in the GEMS, and these are not seen. Moreover, the GEMS are confined to a narrow size range $(0.1-0.5 \mu \mathrm{m})$, whereas some of the enstatite and olivine crystals in the chondritic aggregate IDPs are micrometer-sized. The conditions of formation of silicate glasses and crystals are extensively documented in a vast body of literature on geochemical thermodynamics and igneous and metamorphic petrology. Although the temperature and pressure regimes are well outside those of low-temperature annealing, the underlying thermodynamic constraints are relevant.

If a large fraction of cometary dust consists of solar system condensates, then radial gradients in the temperature, composition, and extent of mixing within the solar nebula should be evident today as differences in the dust chemistry and mineralogy among comets formed in different regions. For example, if the olivine condensed in the hot inner solar nebula and was transported outward or if olivine was created in situ by passing shock waves, then one would expect to see a difference in olivine abundance between Oort cloud comets that formed in the region of the giant planets and Kuiper belt comets that formed beyond 30 AU. To date, no strong 11.2- $\mu$ m olivine peak has been detected in a Kuiper belt comet; however, the cause may be a lack of disaggregated small grains in the coma rather than a lack of silicates. A possible, but weak, 11.2- $\mu$ m peak may be present in the relatively weak silicate features observed in Comets P/Borrelly (Hanner et al., 1996) and P/ Hartley 2 (Crovisier et al., 2000).

Could the crystalline silicates have a presolar origin? Silicate grains are known to condense in O-rich envelopes around evolved stars. Spectral peaks of the $\mathrm{Mg}$-silicates forsterite and enstatite are clearly present in the ISO spectra of some evolved stars (Waters et al., 1996; Molster et al., 2002). Yet signatures of crystalline silicates are absent in spectra of the diffuse interstellar medium (ISM) or molecular clouds. Moreover, the spectra of most young stellar objects show no evidence of crystalline grains. Only in debris disks around young main-sequence objects such as $\beta$ Pictoris (Knacke et al., 1993) and in certain late-stage
Herbig Ae/Be stars that are precursors of $\beta$ Pictoris systems (Waelkens et al., 1996) does one find the spectral peaks of crystalline olivine. For example, the ISO spectrum of the late-stage Herbig Ae/Be star HD100546 is very similar to that of Comet Hale-Bopp (Malfait et al., 1998). These systems are thought to have developed a population of comets that are the source of the dust (e.g., Weissman, 1984; Grady et al., 1997).

Grain destruction in the ISM is an efficient process. Thus, if the comet grains formed in circumstellar outflows from evolved stars, one has to understand how they survived destruction in the ISM and why their spectral signatures are not seen in the ISM or young stellar objects.

Kemper et al. (2001) have produced radiative transfer models to study the visibility of crystalline silicates in midinfrared spectra of circumstellar dust. Because pure crystalline $\mathrm{Mg}$-silicates are very transparent at visible and nearIR wavelengths, they will be colder than Fe-containing amorphous silicates in optically thin regions where grains are heated by exposure to visible/near-IR stellar radiation. Kemper et al. showed that, indeed, a considerable fraction of forsterite and enstatite could be present in such environments without their spectral signatures being visible in emission above the radiation from the warmer dust components. These results should make us cautious about concluding that crystalline silicates are absent in cases where they may simply be "hidden" by their cold temperatures, a point also discussed by Wooden et al. (2000).

Anomalous isotopic enrichments could constitute strong evidence for an interstellar origin of cometary dust material. As described in section 4, analysis techniques have now reached the point where isotopic measurements can be made for individual grains in IDPs, and the first O-isotopic measurements provide tantalizing hints that a few grains do possess nonsolar O-isotopic ratios. However, it is not at all clear that silicate grains formed in the interstellar medium would have anomalous isotopic abundances. Their compositions may well have been homogenized to an average close to solar abundances.

Greenberg (1982) proposed that interstellar silicate grains possess organic refractory mantles as a result of UV photoprocessing in the diffuse interstellar medium following deposition of icy mantles in cold molecular clouds. These submicrometer core/mantle grains, perhaps with an additional icy mantle, were subsequently agglomerated and incorporated into comets. A mass of the organic refractory material comparable to the mass of the silicates satisfied cosmic abundances; in fact, it was argued that organic refractory mantles are a necessary repository of $\mathrm{C}$ to account for its cosmic abundance. The detection of high $\mathrm{D}$ enrichments in the organic refractory material in chondritic IDPs lends strong support to a presolar origin for the organic refractory material. The high $\mathrm{D} / \mathrm{H}$ material often surrounds GEMS and other mineral grains, suggesting that these embedded grains are presolar as well, although the material is more clumpy and irregular rather than a uniform core/mantle morphology. However, the distinction between a population of GEMS with carbonaceous mantles and a popula- 
tion of GEMS within a carbonaceous matrix is difficult to discern in the electron microscope. Organic refractory grains have not been detected spectroscopically in comets. An emission feature at $3.4 \mu \mathrm{m}$ first detected in P/Halley, but also seen in other comets, was initially attributed to organic refractory grains. However, the discovery of methanol in comets and analysis of its infrared bands led to the conclusion that the 3.4- $\mu \mathrm{m}$ feature is due to gas phase methanol and other gaseous species (Bockelée-Morvan et al., 1995; DiSanti et al., 1995). For further discussion concerning the origin of cometary grains, see Wooden (2002).

\section{FUTURE DIRECTIONS}

The past decade has witnessed significant advances in our understanding of the chemistry and mineralogy of cometary dust. However, questions remain about the nature of the refractory organic material, the differing dust properties among comets, and the origins of the various dust components.

The next decade should bring further advances in our knowledge of the composition and origin of cometary dust, including the first comet dust sample return. NASA's Stardust mission, launched in 1999, will collect a dust sample from Comet 81P/Wild 2 in January 2004 and return it to Earth in January 2006. Coupled with the newest analysis tools, the sample will yield isotopic abundance ratios for individual particles, as well as the chemistry and mineralogy of the silicates and other refractory dust components from a comet that probably originated in the Kuiper belt.

ESA's ambitious Rosetta mission, now scheduled for launch in 2004, will rendezvous with a short-period comet. Rosetta carries several instruments to measure the dust mass distribution, composition, and structure, as the spacecraft travels with the comet from aphelion to perihelion (Schwehm and Schulz, 1999).

The midinfrared spectra of Comet Hale-Bopp and astronomical sources from ISO demonstrated the value of 8 45- $\mu \mathrm{m}$ spectroscopy for determining dust composition. SIRTF, the Space Infrared Telescope Facility, and SOFIA, the Stratospheric Observatory for Infrared Astronomy, will allow a number of comets to be observed in this important spectral region. The detection of crystalline silicates in Kuiper belt comets would be particularly significant in showing that crystalline silicate particles were spread throughout the solar nebula.

Finally, improved sensitivity of laboratory techniques for compositional analysis of microscopic samples, such as the nanoSIMS, will offer the opportunity for elemental and isotopic abundances to be investigated in individual submicrometer-sized grains in IDPs and returned cometary samples.

\section{CONCLUSIONS}

In situ sampling of Comet Halley dust, remote infrared spectroscopy, and IDP analyses yield a consistent picture of the composition of cometary dust. Silicates constitute the most abundant material; they are present in both crystal- line and amorphous (or glassy) form and include the mineralogy of both olivine and pyroxene. The crystalline silicates are primarily the $\mathrm{Mg}$-rich minerals forsterite and enstatite, as is the case for circumstellar dust around evolved stars. Carbon is present in approximately cosmic abundance, much of it in an organic refractory component.

We have discussed why the chondritic porous aggregate IDPs are probably from comets. Analysis of their properties complements and substantiates the conclusions drawn from the spectroscopy and from the in situ measurements during the 1986 Halley spacecraft encounters. In particular, IDP analysis confirms that much of the carbonaceous material is in an organic phase, and the detection of high $\mathrm{D} / \mathrm{H}$ ratios implies that at least some of this material is presolar. The D-rich material often surrounds GEMS and other mineral grains, suggesting that these embedded grains are of presolar origin as well. GEMS are the predominant form of noncrystalline silicates. These particles appear to have experienced high radiation dosage in a presolar environment.

The various types of silicate particles do not necessarily have a common origin. While the GEMS are of likely presolar origin, the origin(s) of the crystalline silicates is unclear. If formed as high-temperature condensates or by annealing in the inner solar nebula, radial transport must have been more efficient during the planetesimal accretion phase than some models predict. It is possible that grains were annealed by transient heating from passing shock waves in the solar nebula at $r \leq 10 \mathrm{AU}$. However, the size range and composition of the crystalline silicates in porous aggregate IDPs are not consistent with what one would expect for annealed GEMS. In either case, one would expect crystalline silicates to be less abundant - or absent entirely - in the Kuiper belt comets that formed beyond $30 \mathrm{AU}$.

If the crystalline silicates were already present in the cloud from which the solar nebula formed, then one needs to explain why their spectral signatures are not seen in interstellar dust or in young stellar objects. The very cold temperatures of "clean" Mg-rich silicates is one possible explanation why their spectral features are not seen in emission. Isotopic measurements of individual silicate grains in IDPs and returned comet samples with nanoSIMS techniques may help to clarify their origin.

Although the chondritic aggregate IDPs have given us extremely interesting insight into the nature of probable cometary dust, we do not know the specific source of an individual IDP, nor the selection effects between comet ejection and Earth capture. Thus comet dust sample return and in situ analysis are very important. In the next decade, we can look forward to the Stardust sample return from the short-period Comet 81P/Wild 2 in January 2006 and the encounter of ESA's Rosetta mission with a short-period comet in 2011-2013.

Acknowledgments. The research of M.S.H. was carried out at the Jet Propulsion Laboratory, California Institute of Technology, under contract with the National Aeronautics and Space Administration. J.P.B. acknowledges support from NASA grants NAG5-7450 and NAG5-9797. 


\section{REFERENCES}

Aleon J., Robert F., Chaussidon M., Marty B., and Engrand C. (2002) ${ }^{15} \mathrm{~N}$ excesses in deuterated organics from two interplanetary dust particles (abstract). In Lunar and Planetary Science XXXIII, Abstract \#1397. Lunar and Planetary Institute, Houston (CD-ROM).

Bell K. R., Cassen P. M., Wasson J. T., and Woolum D. S. (2000) The FU Orionis phenomenon and solar nebula material. In: Protostars and Planets IV (V. Mannings et al., eds.), pp. 897926. Univ. of Arizona, Tucson.

Bockelée-Morvan D., Brooke T. Y., and Crovisier J. (1995) On the origin of the 3.2- to 3.6- $\mu \mathrm{m}$ emission features in comets. Icarus, 116, 18-39.

Bockelée-Morvan D., Gautier D., Hersant F., Hure J-M., and Robert F. (2002) Turbulent radial mixing in the solar nebula as the source of crystalline silicates in comets. Astron. Astrophys., 384, 1107-1118.

Boss A. P. (1994) Midplane temperatures and solar nebula evolution (abstract). In Lunar and Planetary Science XXV, p. 149. Lunar and Planetary Institute, Houston.

Boss A. P. (1998) Temperatures in protoplanetary disks. Annu. Rev. Earth Planet. Sci., 26, 53-80.

Boss A. P. (2004) From molecular clouds to circumstellar disks. In Comets II (M. C. Festou et al., eds.), this volume. Univ. of Arizona, Tucson.

Bradley J. P. (1994) Chemically anomalous, preaccretionally irradiated grains in interplanetary dust from comets. Science, 265, 925.

Bradley J. P., Brownlee D. E., and Veblen D. R. (1983) Pyroxene whiskers and platelets in interplanetary dust: Evidence of vapour phase growth. Nature, 301, 473-477.

Bradley J. P., Humecki H. J., and Germani M. S. (1992) Combined infrared and analytical electron microscope studies of interplanetary dust particles. Astrophys. J., 394, 643-651.

Bradley J. P., Keller L. P., Brownlee D. E., and Thomas K. L. (1996) Reflectance spectroscopy of interplanetary dust particles. Meteoritics \& Planet. Sci., 31, 394-402.

Bradley J. P., Keller L. P., Snow T. P., Hanner M. S., Flynn G. J., Gezo J. C., Clemett S. J., Brownlee D. E., and Bowey J. E. (1999) An infrared spectral match between GEMS and interstellar grains. Science, 285, 1716-1718.

Bregman J. H., Campins H., Witteborn F. C., Wooden D. H., Rank D. M., Allamandola L. J., Cohen M., and Tielens A. G. G. M. (1987) Airborne and groundbased spectrophotometry of Comet P/Halley from 5-13 um. Astron. Astrophys., 187, 616-620.

Brucato J. R., Colangeli L., Mennella V., Palumbo P., and Bussoletti E. (1999) Silicates in Hale-Bopp: Hints from laboratory studies. Planet. Space Sci., 47, 773-779.

Brucato J. R., Mennella V., Colangeli L., Rotundi A., and Palumbo P. (2002) Production and processing of silicates in laboratory and in space. Planet. Space Sci., 50, 829-837.

Campins H. and Ryan E. (1989) The identification of crystalline olivine in cometary silicates. Astrophys. J., 341, 1059-1066.

Campins H., Rieke G. H., and Lebofsky M. J. (1982) Infrared photometry of periodic comets Encke, Chernykh, KearnsKwee, Stephan-Oterma, and Tuttle. Icarus, 51, 461-465.

Carrez Ph., Demyk K., Cordier P., Gengembre L., Grimblot J., d'Hendecourt L., Jones A., and Leroux H. (2002) Low-energy helium ion irradiation-induced amorphization and chemical changes in olivine: Insights for silicate dust evolution in the interstellar medium. Meteoritics \& Planet. Sci., 37, 1599-1614.
Chick K. M. and Cassen P. (1997) Thermal processing of interstellar dust grains in the primitive solar environment. Astrophys. J., 477, 398-409.

Clemett S. J., Maechling C. R., Zare R. N., Swan P. D., and Walker R. M. (1993) Identification of complex aromatic molecules in individual interplanetary dust particles. Science, 262, 721.

Colangeli L., Mennella V., Brucato J. R., Palumbo P., and Rotundi A. (1999) Characterization of cosmic materials in the laboratory. Space Sci. Rev., 90, 341-354.

Crovisier J., Brooke T. Y., Hanner M. S., Keller H. U., Lamy P. L., Altieri B., Bockelée-Morvan D., Jorda L., Leech K., and Lellouch E. (1996) The Infrared spectrum of Comet C/1995 O1 (Hale-Bopp) at 4.6 AU from the Sun. Astron. Astrophys., 315, L385-L388.

Crovisier J., Leech K., Bockelée-Morvan D., Brooke T. Y., Hanner M. S., Altieri B., Keller H. U., and Lellouch E. (1997) The spectrum of Comet Hale-Bopp (C/1995 O1) observed with the Infrared Space Observatory at 2.9 astronomical units from the Sun. Science, 275, 1904-1907.

Crovisier J. and 14 colleagues (2000) The thermal infrared spectra of Comets Hale-Bopp and 103P/Hartley 2 observed with the Infrared Space Observatory. In Thermal Emission Spectroscopy and Analysis of Dust, Disks, and Regoliths (M. L. Sitko et al., eds.), pp. 109-117. ASP Conference Series 196.

Dai Z. R. and Bradley J. P. (2001) Iron-nickel sulfides in anhydrous interplanetary dust particles. Geochim. Cosmochim. Acta, 65, 3601-3612.

Day K. L. (1976) Further measurements of amorphous silicates. Astrophys. J., 210, 614-617.

Day K. L. (1981) Infrared extinction of amorphous iron silicates. Astrophys. J., 368, 110-112.

Demyk K., Carrez Ph., Leroux H., Cordier P., Jones A. P., Borg J., Quirico E., Raynal P. I., and d'Hendecourt L. (2001) Structural and chemical alteration of crystalline olivine under low energy $\mathrm{He}^{+}$irradiation. Astron. Astrophys., 368, L38-L41.

DiSanti M. A., Mumma M. J., Geballe T. R., and Davies J. K. (1995) Systematic observations of methanol and other organics in Comet P/Swift-Tuttle. Icarus, 116, 1-17.

Dorschner J., Begemann B., Henning Th., Jäger C., and Mutschke H. (1995) Steps toward interstellar silicate mineralogy II. Study of $\mathrm{Mg}$-Fe-silicate glasses of variable composition. Astron. Astrophys., 300, 503-520.

Engrand C., McKeegan K. D., Leshin L. A., Bradley J. P., and Brownlee D. E. (1999) Oxygen isotopic composition of interplanetary dust particles: ${ }^{16} \mathrm{O}$-excess in a GEMS-rich IDP (abstract). In Lunar and Planetary Science XXX, Abstract \#1690. Lunar and Planetary Institute, Houston (CD-ROM).

Fabian D., Jäger C., Henning Th., Dorschner J., and Mutschke H. (2000) Steps toward interstellar silicate mineralogy $\mathrm{V}$. Thermal evolution of amorphous magnesium silicates and silica. Astron. Astrophys., 364, 282-292.

Flynn G. J., Keller L. P., Jacobsen C. and Wirick S. (1999) Organic carbon in cluster IDPs from the L2009 and L2011 collectors (abstract). In Lunar and Planetary Science $X X X$, Abstract \#1091. Lunar and Planetary Institute, Houston (CDROM).

Flynn G. J., Keller L. P., Jacobsen C., Wirick S. and Miller M. A. (2000) Interplanetary dust particles as a source of pre-biotic organic matter on the Earth (abstract). In Lunar and Planetary Science XXXI, Abstract \#1409. Lunar and Planetary Institute, Houston (CD-ROM).

Flynn G. J., Feser M., Keller L. P., Jacobsen C., and Wirik S. 
(2001) Carbon-XANES and oxygen-XANES measurements on interplanetary dust particles: A preliminary measurement of the $\mathrm{C}$ to $\mathrm{O}$ ratio in the organic matter in a cluster IDP (abstract). In Lunar and Planetary Science XXXII, Abstract \#1603. Lunar and Planetary Institute, Houston (CD-ROM).

Fomenkova M., Kerridge J., Marti K., and McFadden L. (1992) Compositional trends in rock-forming elements of Comet Halley dust. Science, 258, 266-269.

Fomenkova M. N., Chang S., and Mukhin L. M. (1994) Carbonaceous components in the Comet Halley dust. Geochim. Cosmochim. Acta, 58, 4503-4512.

Galdemard P., Lagage P. O., Dubreuil D., Jouan R., Masse P., Pantin E., and Bockelée-Morvan D. (1999) Mid-infrared spectro-imaging observations of Comet Hale-Bopp. Earth Moon Planets, 78, 271-277.

Gehrz R. D., Ney E. P., Piscitelli J., Rosenthal E., and Tokunaga A. T. (1989) Infrared photometry and spectroscopy of Comet P/Encke in 1987. Icarus, 80, 280-288.

Glaccum W., Moseley S. H., Campins H., and Loewenstein R. F. (1987) Airborne spectrophotometry of P/Halley from 20 to 65 microns. Astron. Astrophys., 187, 635-638.

Grady C. A., Sitko M. L., Bjorkman K. S., Perez M. R., Lynch D. K, Russell R. W., and Hanner M. S. (1997) The star-grazing extrasolar comets in the HD100546 system. Astrophys. J., 483, 449-456.

Greenberg J. M. (1982) What are comets made of? A model based on interstellar dust. In Comets (L. L. Wilkening, ed.), pp. 131163. Univ. of Arizona, Tucson.

Grün E. and 23 colleagues (2001) Broadband infrared photometry of Comet Hale-Bopp with ISOPHOT. Astron. Astrophys., 377, 1098-1118.

Hallenbeck S. L., Nuth J. A., and Daukantas P. L. (1998) Midinfrared spectral evolution of amorphous magnesium silicate smokes annealed in vacuum: Comparison to cometary spectra. Icarus, 131, 198-209.

Hallenbeck S. L., Nuth J. A., and Nelson R. N. (2000) Evolving optical properties of annealing silicate grains: From amorphous condensate to crystalline mineral. Astrophys. J., 535, 247-255.

Hanner M. S. and Hayward T. L. (2003) Infrared observations of Comet 81P/Wild 2 in 1997. Icarus, 161, 164-173.

Hanner M. S., Newburn R. L., Gehrz R. D., Harrison T., Ney E. P., and Hayward T. L. (1990) The Infrared spectrum of Comet Bradfield (1987s) and the silicate emission feature. Astrophys. J., 348, 312-321.

Hanner M. S., Lynch D. K., and Russell R. W. (1994a) The 8-13 micron spectra of comets and the composition of silicate grains. Astrophys. J., 425, 274-285.

Hanner M. S., Hackwell J. A., Russell R. W., and Lynch D. K. (1994b) Silicate emission feature in the spectrum of Comet Mueller 1993a. Icarus, 112, 490-495.

Hanner M. S., Lynch D. K., Russell R. W., Hackwell J. A., and Kellogg R. (1996) Mid-infrared spectra of Comets P/Borrelly, $\mathrm{P} /$ Faye, and P/Schaumasse. Icarus, 124, 344-351.

Hanner M. S., Gehrz R. D., Harker D. E., Hayward T. L., Lynch D. K., Mason C. G., Russell R. W., Williams D. M., Wooden D. H., and Woodward C. E. (1999) Thermal emission from the dust coma of Comet Hale-Bopp and the composition of the silicate grains. Earth Moon Planets, 79, 247-264.

Harker D. E. and Desch S. J. (2002) Annealing of silicate dust by shocks at 10 AU. Astrophys. J. Lett., 565, L109-L112.

Harker D. E., Wooden D. H., Woodward C. E., and Lisse C. M.
(2002) Grain properties of Comet C/1995 O1 (Hale-Bopp). Astrophys. J., 580, 579-597.

Hayward T. L., Hanner M. S., and Sekanina Z. (2000) Thermal infrared imaging and spectroscopy of Comet Hale-Bopp (C/ 1995 O1). Astrophys. J., 538, 428-455.

Herter T., Campins H., and Gull G. E. (1987) Airborne spectrophotometry of P/Halley from 16 to 30 microns. Astron. Astrophys., 187, 629-631.

Jäger C., Molster F. J., Dorschner J., Henning Th., Mutschke H., and Waters L. B. F. M. (1998) Steps toward interstellar silicate mineralogy IV. The crystalline revolution. Astron. Astrophys., 339, 904-916.

Jessberger E. (1999) Rocky cometary particulates: Their elemental, isotopic, and mineralogical ingredients. Space Science Rev., 90, 91-97.

Jessberger E. K., Christoforidis A., and Kissel J. (1988) Aspects of the major element composition of Halley's dust. Nature, 332, 691-695.

Keller L. P. and Messenger S. (1997) Reflectance spectroscopy of deuterium-rich cluster IDPs (abstract). In Lunar and Planetary Science XXVIII, pp. 705-706. Lunar and Planetary Institute, Houston.

Keller L. P., Messenger S., and Bradley J. P. (2000) Analysis of a deuterium-rich interplanetary dust particle (IDP) and implications for presolar material in IDPs. J. Geophys. Res., 105, 10397-10402.

Keller L. P. and 10 colleagues (2002) Identification of iron sulphide grains in protoplanetary disks. Nature, 417, 148-150.

Kemper F., Waters L. B. F. M., de Koter A., and Tielens A. G. G. M. (2001) Crystallinity versus mass loss rate in asymptotic giant branch stars. Astron. Astrophys., 369, 132-141.

Kissel J. and 22 colleagues (1986a) Composition of Comet Halley dust particles from Vega observations. Nature, 321, 280-282.

Kissel J. and 18 colleagues (1986b) Composition of Comet Halley dust particles from Giotto observations. Nature, 321, 336-338.

Klock W., Thomas K. L., McKay D. S., and Palme H. (1989) Unusual olivine and pyroxene composition in interplanetary dust and unequilibrated ordinary chondrites. Nature, 339, 126128.

Knacke R. F., Fajardo-Acosta S. B., Telesco C. M., Hackwell J. A., Lynch D. K., and Russell R. W. (1993) The silicates in the disk of $\beta$ Pictoris. Astrophys. J., 418, 440-450.

Koike C. and Tsuchiyama A. (1992) Simulation and alteration for amorphous silicates with very broad bands in infrared spectra. Mon. Not. R. Astron. Soc., 255, 248-254.

Koike C., Shibai H., and Tuchiyama A. (1993) Extinction of olivine and pyroxene in the mid- and far-infrared. Mon. Not. R. Astron. Soc., 264, 654-658.

Lawler M. E. and Brownlee D. E. (1992) CHON as a component of dust from Comet Halley. Nature, 359, 810-812.

Lawler M. E., Brownlee D. E., Temple S., and Wheelock M. M. (1989) Iron, magnesium, and silicon in dust from Comet Halley. Icarus, 80, 225-242.

Lynch D. K., Russell R. W., Campins H., Witteborn F. C., Bregman J. D., Rank D. W., and Cohen M. C. (1989) 5-13 $\mu \mathrm{m}$ observations of Comet Wilson 19861. Icarus, 82, 379-388.

Lynch D. K., Russell R. W., Hackwell J. A., Hanner M. S., and Hammel H. B. (1992) 8-13 $\mu$ m spectroscopy of Comet Levy 1990 XX. Icarus, 100, 197-202.

Malfait K., Waelkens C., Waters L. B. F. M., Vandenbussche B., Huygen E., and de Graauw M. S. (1998) The spectrum of the young star HD100546 observed with the Infrared Space Ob- 
servatory. Astron. Astrophys., 332, L25-L28.

Meier R. and Owen T. C. (1999) Cometary deuterium. Space Sci. Rev., 90, 33-43.

Messenger S. (2000) Identification of molecular-cloud material in interplanetary dust particles. Nature, 404, 968-971.

Messenger S., Keller L. P., Stadermann F. J., Walker R. M., and Zinner E. (2003) Samples of stars beyond the solar system: Silicate grains in interplanetary dust. Science, 300, 105-108.

Molster F. J., Waters L. B. F. M., Tielens A. G. G. M., and Barlow M. J. (2002) Crystalline silicate dust around evolved stars I. The sample stars. Astron. Astrophys., 383, 184-221.

Nier A. O. and Schlutter D. J. (1993) The thermal history of interplanetary dust particles collected in the Earth's stratosphere. Meteoritics, 28, 675-581.

Sandford S. A. and Walker R. M. (1985) Laboratory infrared transmission spectra of individual interplanetary dust particles from 2.5 to 25 microns. Astrophys. J., 291, 838-851.

Schulze H., Kissel J., and Jessberger E. (1997) Chemistry and mineralogy of Comet Halley's dust. In From Stardust to Planetesimals (Y. J. Pendleton and A. G. G. M. Tielens, eds.), pp. 397-414. ASP Conference Series 122, Astronomical Society of the Pacific, San Francisco.

Schwehm G. and Schulz R. (1999) Rosetta goes to Comet Wirtanen. Space Sci. Rev., 90, 313-319.
Stephens J. R. and Russell R. W. (1979) Emission and extinction of ground and vapor-condensed silicates from 4 to 14 microns and the 10 micron silicate feature. Astrophys. J., 228, 780-786.

Thomas K. L., Blanford G. E., Keller L. P., Klock W., and McKay D. S. (1993) Carbon abundance and silicate mineralogy of anhydrous interplanetary dust particles. Geochim. Cosmochim. Acta, 57, 1551-1566.

Waters L. B. F. M. and 36 colleagues (1996) Mineralogy of oxygen-rich dust shells. Astron. Astrophys., 315, L361-L364.

Waelkens C. and 20 colleagues (1996) SWS observations of young main-sequence stars with dusty circumstellar disks. Astron. Astrophys., 315, L245-248.

Weissman P. R. (1984) The Vega particulate shell: Comets or asteroids. Science, 224, 987.

Wooden D. H. (2002) Comet grains: Their IR emission and their relation to ISM grains. Earth Moon Planets, 89, 247-287.

Wooden D. H., Harker D. E., Woodward C. E., Koike C., Witteborn F. C., McMurtry M. C., and Butner H. M. (1999) Silicate mineralogy of the dust in the inner coma of Comet C/ 1995 O1 (Hale-Bopp) pre- and post-perihelion. Astrophys. J., 517, 1034-1058.

Wooden D. H., Butner H. M., Harker D. E., and Woodward C. E. (2000) Mg-rich silicate crystals in Comet Hale-Bopp: ISM relics or solar nebula condensates? Icarus, 143, 126-137. 